\section{EMBRYRIDDLE \\ Aeronautical University}

SCHOLARLY COMMONS
International Journal of Aviation, Aeronautics, and Aerospace

\title{
Impact of Electronic Flight bag (EFB) on Single Pilot Performance and Workload
}

\author{
Saravanan Suppiah \\ Embry-Riddle Aeronautical University, SUPPIAHS@my.erau.edu \\ Dahai Liu \\ Embry-Riddle Aeronautical University - Daytona Beach, liu89b@erau.edu \\ Sang-A Lee \\ LEES98@my.erau.edu \\ Andrew Dattel \\ andy.dattel@erau.edu \\ Dennis Vincenzi Ph.D. \\ Embry-Riddle Aeronautical University - Worldwide, vincenzd@erau.edu
}

Follow this and additional works at: https://commons.erau.edu/ijaaa

Part of the Applied Behavior Analysis Commons, and the Human Factors Psychology Commons

\section{Scholarly Commons Citation}

Suppiah, S., Liu, D., Lee, S., Dattel, A., \& Vincenzi, D. (2020). Impact of Electronic Flight bag (EFB) on Single Pilot Performance and Workload. International Journal of Aviation, Aeronautics, and Aerospace, 7(4).

https://doi.org/10.15394/ijaaa.2020.1531

This Article is brought to you for free and open access by the Journals at Scholarly Commons. It has been accepted for inclusion in International Journal of Aviation, Aeronautics, and Aerospace by an authorized administrator of Scholarly Commons. For more information, please contact commons@erau.edu. 
As the need for flying keeps increasing, the requirement for aircraft to operate efficiently and yet safely in demanding flight environments has become necessary (Salas \& Marino, 2010). Aircraft manufacturers continue to build highly automated aircraft (i.e., head-up display with night vision), find ways to automate flight instruments, and equip the flight crew with recent technologies to facilitate the demands of aircraft operations. These technologies are not only available to commercial aircraft but are also increasingly adopted by pilots flying general aviation (GA) aircraft (Chandra \& Kendra, 2009). One of the popular automation technologies is the Electronic Flight Bag (EFB).

The name EFB took its roots from the traditional flight bags carried by pilots, which contained numerous numbers of paper-based flight checklist, aeronautical charts, weather charts, and volumes of manuals (Ates, 2017). These documents (i.e., navigational charts, manuals, and advisories) are vital resources for flight operations, especially during critical phases of flight (Babb, 2017b). The pilots need to access them quickly in flight without compromising flight safety. These charts are usually clipped onto the control yolks for easy visibility (Babb, 2017b). If these charts accidentally fall on the cockpit floor, it can be difficult to retrieve them as the cockpit spaces are usually tiny. They are also prone to wear and tear (Cahill \& Donald, 2006). The earliest adopters of EFB were the FedEx pilots in the 1990s (Babb, 2017b). Their flight deck was equipped with laptop computers, referred to as Airport Performance Laptop Computers (APLCs) (Babb, 2017a).

The APLC can perform aircraft performance calculations, for example, determining aircraft's runway stopping distance or calculating the maximum takeoff weight of the aircraft (MTOW) (Babb, 2017a). The arrival of hand-held devices with touch screen capabilities encouraged aircraft manufacturers to collaborate with software developers specializing in flight management software such as Jeppesen, Foreflight, and Garmin to develop solutions to migrate paperbased forms to electronic copies which can be easily accessed by pilots using handheld devices (Ohme, 2014). Soon software developers were able to migrate the paper versions of aircraft documents and incorporate aircraft performance applications into an electronic platform that can be viewed on handheld tablets (Babb, 2017a).

Over the years, the utility of EFB and its use in the aircraft cockpit have been well received by pilots (Haddock \& Beckman, 2015). One of the key advantages of using EFB is that it replaces the traditional use of paper-based aeronautical documents with electronic versions (Haddock \& Beckman, 2015). Pilots are now able to access and view real-time relevant aeronautical documents (i.e., sectional charts, weather charts, or safety circulars) in high resolution using EFB (Haddock \& Beckman, 2015). The shift from paper to electronic copies allows pilots to use the EFB as one-stop access to managing all aircraft documents. The 
EFB serves as an intermediate role between the pilot and the airlines by providing transparency of aircraft operational records (i.e., pilot records on system issues in air and on the ground). It provides an avenue for airlines to digitalize pilot task processes or validations, which was traditionally done in a paper (i.e., go around checks, fuel loading documents) (Haddock \& Beckman, 2015). The EFB can also prevent aircraft documents from being lost.

Another key advantage of using EFB is that it offers high-speed internet connectivity for the pilot to send and receive timely feedback about the aircraft's health and flight safety matters to the aircraft stockholders (Ates, 2017; Chandra, 2003). One of the critical advantages of EFB is its ability to relieve pilots from handling various paper-based charts and checklists while operating the aircraft (Babb, 2017a). With an EFB, pilots can view high-resolution sectional charts, approach charts, and various aeronautical documents that are essential for the operation of the flight. Furthermore, with the incorporation of Global Position Systems (GPS) in EFB, pilots can view moving airfield maps, which can reduce the workload of the pilots (Babb, 2017a).

However, just like any other automation technologies, the use of EFB in flight has also raised several safety concerns. For example, 37 flight safety incidents and accidents were reported by the U.S. Department of Transport at the initial EFB implementation period between 1995 to 2006. Some of these events include runway incursion, spatial deviation, incorrect weight, and balance computation (Chandra \& Kendra, 2009).

These accidents reiterate the point that the need to pay careful attention to human factor considerations involved between the man-machine interaction in a highly automated environment must be addressed (Joslin, 2013). Some of the negative impacts of EFB can be attributed to the automation biasness, impact of visual perception, and workload issues.

Automation bias is the use of automation as a heuristic substitute to attentively gather and process data, that often leads to the error of omission and commission (Parasuraman, Sheridan, \& Wickens, 2008). For example, the operator does not notice that the EFB software fails to notify the user that the push notification is turned off (German \& Rhodes, 2016). A factor that could influence automation bias is the social loafing attitude of human operators who regard themselves as being less responsible, as the system's performance is expected to function erroneously (Endsley, 1999). Alternatively, it could be due to complacency that occurs when the operator fails to monitor the automated information from the diversion of attention that may result in a loss in situation awareness (German \& Rhodes, 2016). The impact of EFB on the human operator could be further analyzed in terms of human limitations such as perception, attention, and memory (Salas \& Mourino, 2010). 
On the other hand, it can also be argued that the supervision of EFB when flying could add to the existing pilot workload and may impact the overall workload, especially during critical phases of flight with extreme time pressures (i.e., unexpected deviation or weather conditions) (Babb, 2017a). Several studies about workload indicated that human operators experience newer hazards in an automated setting (Archer, Keno, \& Kwon, 2012; Salas \& Marino, 2010). Interestingly it is further explained that the expected work reduction from automation may transform to other means of added workload to the human operator in the future operations of the system. For example, one of the safety issues reported in the Aviation Safety Reporting System (ASRS) was that the pilots had problems zooming and panning the contents in the EFB to a legible level (ASRS, 2018). The pilots were concerned about missing some critical pieces of the information in the EFB, which was necessary to navigate the flight safely (ASRS, 2018). This incident clearly shows that the troubleshooting attempts by pilots outside the perimeter of their primary tasks not only consumes their time, but it also becomes an additional mental load to the existing workload (Archer et al., 2012). Thus, it can be said that the use of EFB during critical phases of flight, such as the approach or deviation, can be detrimental to flight safety (Archer et al., 2012).

With the EFB relatively new in the aviation field, the understanding of its impact on pilot performance and workload is still limited. The study investigated the effect of EFB on the pilot performance and perceived workload during approach (i.e., expected and unexpected approaches) to provide greater insight into the influence of EFB on the GA pilots.

\section{Research Approach}

\section{Method}

This study investigated the impact on the pilot workload based on the use of EFB and paper navigational charts during the approach. In order to measure the workload experienced by the pilot, the National Aeronautics and Space Administration Task Load Index (NASA-TLX) was used after each flight scenario. Before the research study, permission to conduct the research was applied to the Institutional Review Board (IRB) at Embry-Riddle Aeronautical University, and it was granted.

Design. The study has two independent variables (approach, display) and one dependent variable (workload). Each of the independent variables has two levels; the approach factor with levels (expected, unexpected) and the display factor with levels (EFB, paper). The experiment for the study was based on withinsubjects 2 x 2 (Approach [expected, unexpected] x Display [EFB, paper]) factorial design using Analysis of Variance (ANOVA). In the simulator, each participant flew four flight scenarios. In each of the flight scenarios, the participant flew to a designated runway from a 3 nautical mile approach with an (a) EFB with expected 
approach, (b) EFB with unexpected approach, (c) paper with expected approach and (d) paper with unexpected approach. To control for order effect, a counterbalancing technique using Latin Square was applied. An alpha value of 5\% was used to determine significance.

Procedures. Once the participants arrived, they were greeted and briefed regarding the purpose and safety risks of the study involved in the experiment. The participants then received the informed consent form to review and approve before going ahead with their participation in the experiment. Once the participants had signed the informed consent form, they proceeded to the flight simulator. At the Elite-P1 135 Basic Aviation Training Device (BATD) simulator, the participants were briefed about the critical flight controls that to be used to fly the aircraft (i.e., control stick, flaps, rudder, and breaks). The critical flight instruments observed by the pilot in the simulator during the flight was the airspeed indicator and altitude meter.

The researchers took the role of an air traffic controller (ATC) to instruct the participant to fly the desired air routes for each of the four flight scenarios. Once the pilot was seated at the simulator, a pre-flight instruction for each flight scenario was given. The instruction includes the call sign for the Cessna 172 Skyhawk aircraft as Riddle141, the destination airport code, the initial approach distance at the start of the flight, which was 3 nautical miles straight in to the runway and a flight safety message. When a participant is finished with a scenario, he or she proceeded to complete the NASA-TLX for the workload experienced.

Once the NASA-TLX was completed, the participant moved towards completing the next scenario based on the order of approach scenarios as determined by the Latin Square, shown in Table 1. In each scenario, the participant received an EFB or a paper navigational charts to fly the aircraft to a designated airport runway. All four flight scenarios were based on the Visual Flight Rule (VFR) approach. Each participant was compensated $\$ 10$ for their time.

Table 1

\begin{tabular}{lllll}
\multicolumn{2}{l}{ Order of Flight } & Scenarios Tested & & \\
\hline Participant & Scenario 1 & Scenario 2 & Scenario 3 & Scenario 4 \\
\hline 1 & Expected Paper & Unexpected & Expected & Unexpected EFB \\
& Paper & EFB & \\
2 & Unexpected & Unexpected EFB & Expected Paper & $\begin{array}{l}\text { Expected } \\
\text { EFB }\end{array}$ \\
& Paper & & & Expected Paper \\
3 & Unexpected EFB & Expected & Unexpected & \\
& Expected & EFB & Paper & Unexpected \\
4 & EFB & Expected Paper & Unexpected EFB & Paper \\
& & & & \\
\hline
\end{tabular}


Expected scenario and flight path. In the expected scenario, the participant flew the aircraft to Front Range Airport (FTG) from a 3 nautical mile approach and landed on Runway 08. Once the participant has read the pre-flight instruction for the expected approach, an EFB or paper charts were provided depending on the order of the scenario shown in Table 1 . The researcher then loaded the expected scenario into the simulator and took the role of the ATC to provide necessary navigational instructions to the participant to fly the aircraft.

The first instruction from the ATC to the participant is to maintain 6500 feet from a 3 nautical mile approach to FTG and advise ATC when the airport is in sight. Once the ATC received a call back from the participant confirming the airport is in sight, the ATC gave clearance to land on runway 08. Once the aircraft was landed, the scenario was completed. The participant then proceeded to fill up the NASA-TLX. The EFB chart is shown in Figure 1, and the paper chart is shown in Figure 2.

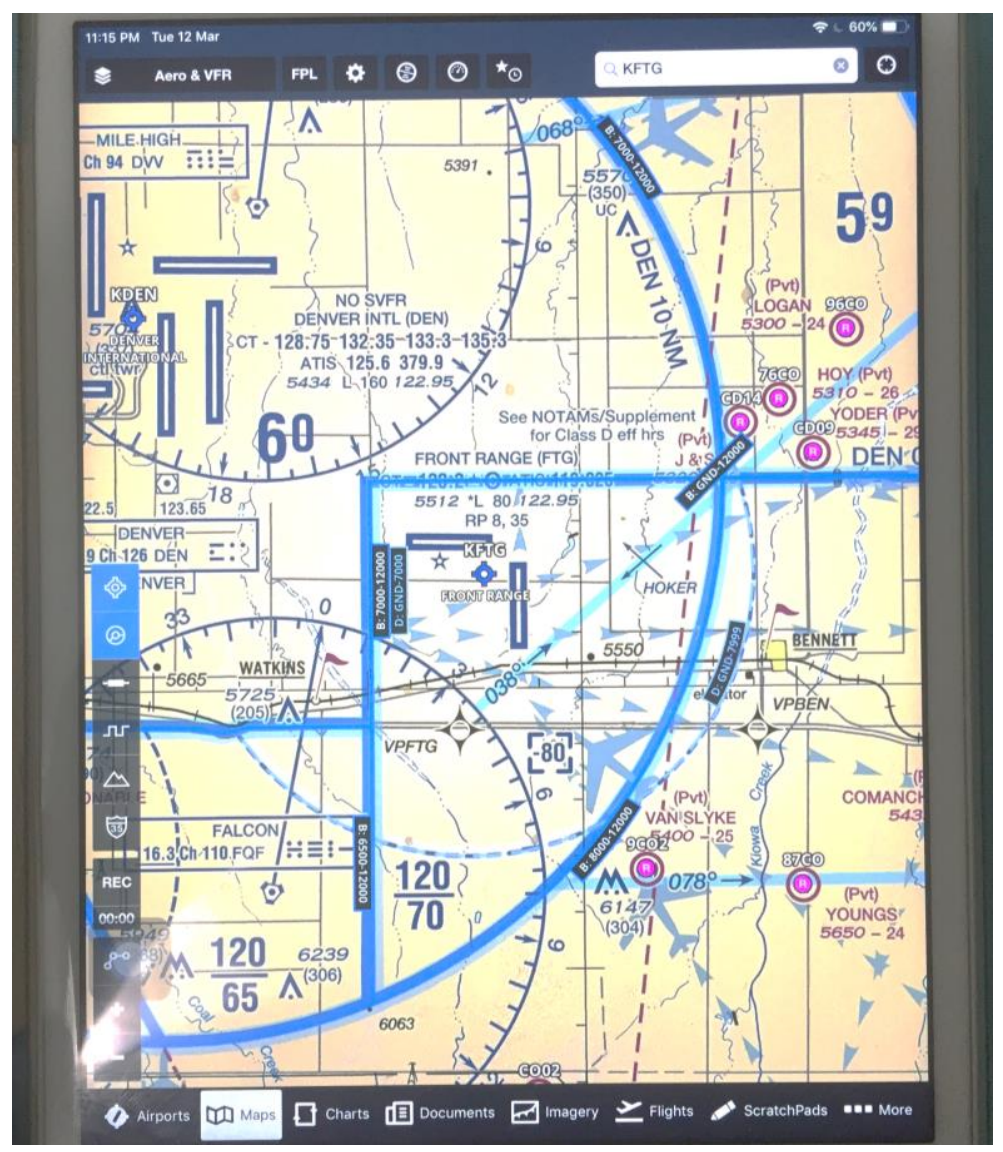

Figure 1. Expected and unexpected VFR sectional chart on paper. 


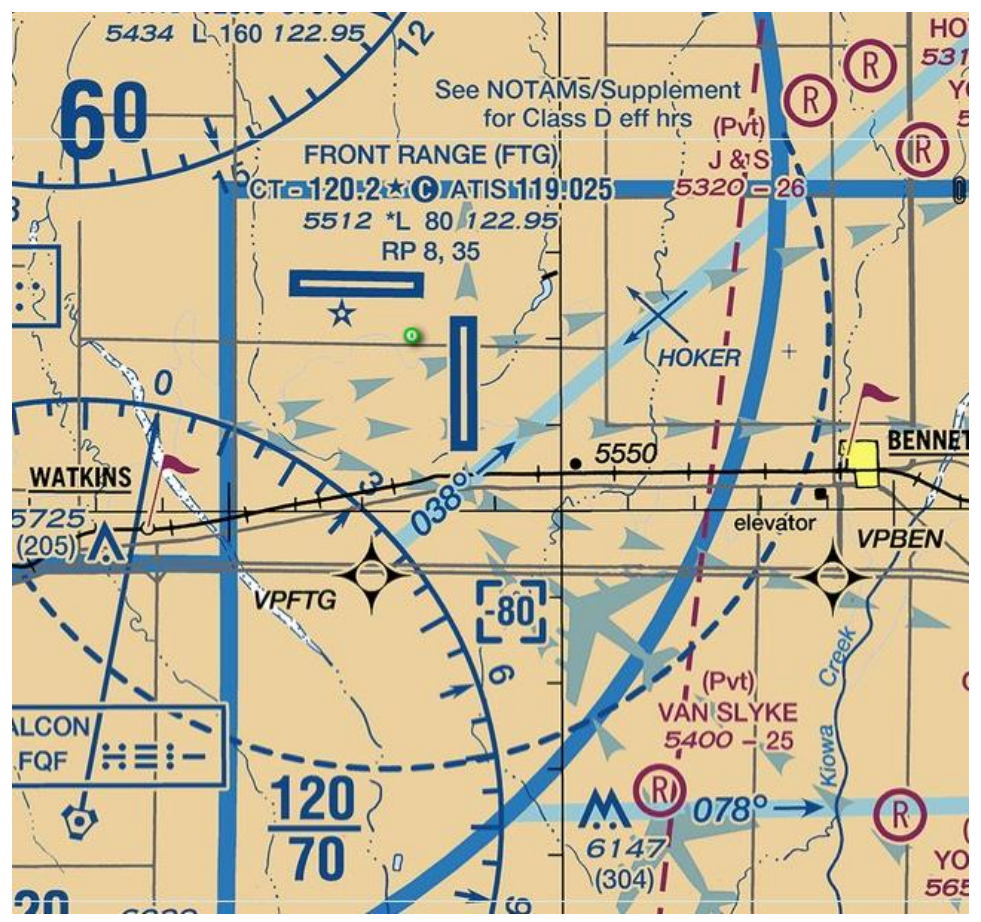

Figure 2. Expected and unexpected VFR sectional chart on EFB.

Unexpected scenario and flight path. In the unexpected scenario, after reading the pre-flight instructions, the participant flew the aircraft to Front Range Airport (FTG) from a 3 nautical mile approach. While on approach to runway 8, the participant was instructed by ATC to take a diversion to land on the adjacent runway 35 due to traffic. Once the participant has read the pre-flight instruction for the unexpected approach, an EFB or paper charts were provided based on the order of the scenario shown in Table 1. The researcher then loaded the unexpected scenario into the simulator. Once the scenario was initiated on the screen, the researcher quickly took the role of the ATC to provide the necessary navigational instructions to the participant to fly the aircraft. The first instruction from the ATC to the participant was to maintain 6500 feet from a 3 nautical mile approach to FTG and make straight in for runway 8 and advice when the airport is in sight. Once the ATC received the call back from the participant that the airport is in sight, the clearance to land on runway 8 was given.

While on approach to runway 8 , the ATC suddenly instructs the participant to divert the aircraft due to traffic on runway 8 and climb to 6500 feet. ATC then instructs the participant to fly right downwind and land on runway 35 instead. Once the participant has landed on runway 35 , the unexpected scenario was completed. The participant then proceeds to complete the NASA-TLX. 
NASA-TLX. In the NASA-TLX, the participants were presented with the six workload factors; mental demand, physical demand, temporal demand, overall performance, effort, and frustration. A detailed description of the six workload factors was also given to them. After reading the description, the participant marked the level of workload experienced for the scenario. They flew from a scale of 0 to 100 for each of the workload factors.

\section{Apparatus and Materials}

The Elite-P1 135 BATD simulator was used to conduct the experiment. This is a low fidelity flight simulator with similar flight controls of Cessna 172 Skyhawk aircraft, which is commonly flown by the pilot students in Embry-Riddle Aeronautical University (ERAU). For each flight scenario, the participant flew an expected or unexpected approach with an EFB or with paper charts. In the scenarios with paper, the participant was given a hard copy of the VFR sectional chart of the airport and the runway map, while for EFB scenarios, the participant received an iPad with ForeFlight software.

The virtual flight environment for the scenarios was delivered using the $\mathrm{X}$ Plane 11 flight simulator software. It is developed by a virtual reality (VR) gaming company called Laminar Research. The X-Plane 11 is suggested as having a robust VR simulation capability, in par with similar professional flight simulator software in the industry (X Plane 11, 2019). Furthermore, X-Plane 11 offers the Cessna 172 Skyhawk cockpit instruments and environments suitable to fly the four flight scenarios to conduct the experiment. Figure 3 shows the simulator setting with $\mathrm{X}$ Plane 11 when in use for the experiment. To ascertain the reliability of the simulator and the X Plane 11 software for this study, the researcher consulted a flight instructor as a subject matter expertise (SME) and conducted a trial experiment.

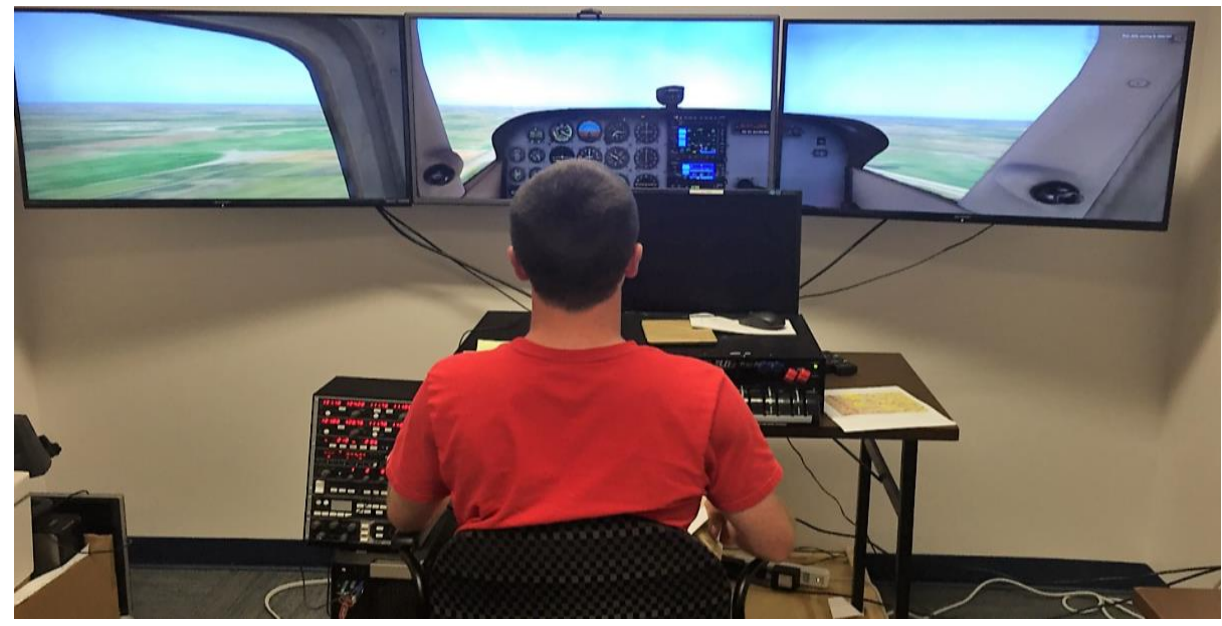

Figure 3. Elite-P1 135 BATD simulator setting with X-Plane 11. 


\section{Population and Sample}

The study required 16 participants with a minimum attainment of private pilot license (PPL). The researcher recruited the participants from the pool of student pilots and instructors from ERAU at Daytona Beach. The source of communication for the recruitment was done via electronic mail and paper flyers. To select the appropriate participants, the researcher used the Google survey to develop a demographic questionnaire. The questionnaire included the participants' names, gender, age, pilot license, flight hours attained. Besides, a flyer was posted around the ERAU campus. To expedite the recruitment, the researcher also communicated with the ERAU flight instructor department to reach out to student pilots. As a token of appreciation, the participants who completed the experiment were given a $\$ 10.00$ Starbucks gift card.

\section{Treatment of the Data}

Each of the scales in the NASA-TLX is shown as a $12-\mathrm{cm}$ line divided into 20 equal intervals. The 21 vertical tick marks on each of the scales divide the scale from 0 to 100 with an increment of 5 .

The participants marked the workload level experienced from a scale of 0 to 100 in increments for the workload factors experienced either with the use of EFB or paper charts during the expected and unexpected scenarios. The overall workload scores obtained were then recorded into the Statistical Package for the Social Sciences (SPSS) statistical software in the researcher computer to test the research hypothesis. To maintain the confidentiality of the participants, the laptop was password protected and only accessible to the researcher. The workload score for each flight scenario was collected using a paper copy of the NASA-TLX.

\section{Results}

Once the workload data were collected, they were organized based on their variables and entered in the SPSS statistical software to test the three-research hypothesis. A $2 \times 2$ within-subjects ANOVA with repeated measures was done in SPSS. The following summarizes the results obtained from the study.

The study was conducted with 16 participants. In terms of flight hours, $62.5 \%$ of the participants had 101-201 hours of flight experience. The average workload scores for EFB $(M=38.91, S D=20.10)$ was higher than paper $(M=$ $37.62, S D=21.08$ ).

Effect of approach. With the alpha level set at .05, a within-subject twoway factorial ANOVA showed a significant main effect for approach $F(1,15)=$ 28.22, $p<.001,\left(\eta_{\mathrm{P}}^{2}=.653\right)$. Therefore, the null hypothesis was rejected. The average workload scores for unexpected approach $(M=47.41, S D=21.21)$ was significantly higher than the workload scores for the expected approach $(M=29.11$, $S D=18.67)$. The effect size is large; thus, it can be concluded that $65.3 \%$ of the variability in the pilot workload scores can be explained by the levels of approach 
(expected and unexpected) being tested. The average workload for the Expected approach was higher than the Unexpected approach. The mean differences between these two approaches is illustrated in Figure 4.

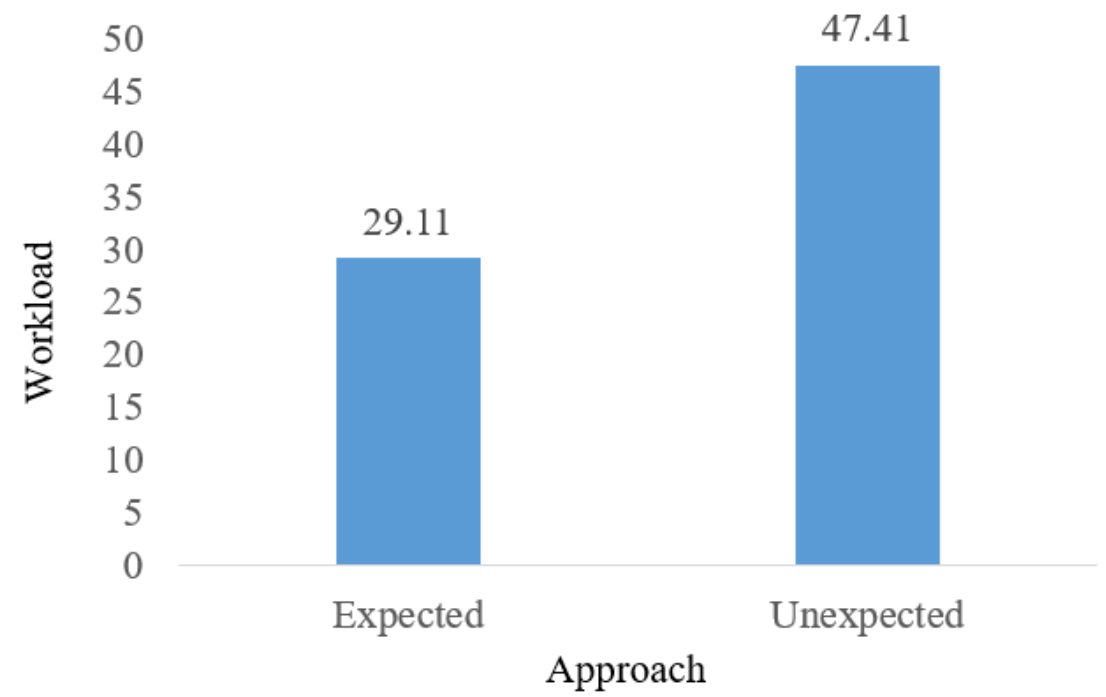

Figure 4. Average workload scores for expected and unexpected approach.

Effect of Display. ANOVA did not show a significant main effect for display $F(1,15)=.091, p>.05,\left(\eta_{\mathrm{P}}^{2}=.006\right)$. The average workload scores for EFB $(M=38.91, S D=20.10)$ was not significantly higher than the average workload scores for paper charts $(M=37.62, S D=21.08)$. As the effect size is small, it can be concluded that only $0.6 \%$ of the variability in the workload scores can be explained by the levels of display (EFB and Paper) being tested.

Interaction Effect. With the alpha level set at .05, the interaction between approach and display was not significant $F(1,15)=81.72, p>.05,\left(\eta_{\mathrm{P}}^{2}=.029\right)$. The effect size is medium; only $29 \%$ of the variability in pilot workload scores can explain the interaction between the levels of approach and display. In the next section, the implications of the findings are discussed.

\section{Pilot Workload Effect on Approach}

\section{Discussion}

This test is to determine whether there is a significant main effect on approach. The test aims to statistically find whether the two levels of approach have a significant impact on the pilot workload. This test is essential for the workload study as the literature suggests that pilots are subjected to a high workload during the approach phase in flight. The researcher was expecting to find a significant effect on the approach. That is, the workload would be higher during unexpected 
scenarios than in expected scenarios. The results of the study indicate that the main effect of the approach was statistically significant. The results also showed that the participants on an unexpected approach experienced a higher workload compared to the expected approach regardless of whether they were using EFB or paper charts. This reiterates the point that the increase in pilot workload due to the increased task demands during the approach persists. The following discusses some of the reasons for this result.

Pilot experience. One key factor that could have contributed to this result is the number and level of experience of the participants involved in this study. For example, based on the demographics of student pilots involved in the study, it is observed that the number of participants with a private pilot license (PPL) was higher than the participants with the commercial pilot license (CPL). This leads to suggest that less flight experience (i.e., flight hours) by participants holding PPL could have contributed to the higher level of workload measured for the unexpected approach.

Unexpected scenario. Another factor for the result could have occurred due to the nature of the task demands required to complete the unexpected approach. The unexpected task scenario involves participants suddenly detour from their original approach from runway 8 to the adjacent runway 35 . This requires the participant to pull the aircraft up to 6500 feet to maintain altitude and re-look at the sectional charts and airport map for runway 35 while flying. Since this is a VFR flight, the participants must look at the simulator monitor and the flight charts to determine their position in the air while looking for runway 35. Furthermore, these tasks were done while communicating with the ATC. Thus, it can be said that the increasing task demands involved in the unexpected scenario could have influenced the pilots' workload.

\section{Pilot Workload Effect on Display}

This test was done to determine whether there is a significant main effect for display. This is to statistically determine whether the two levels of display (paper charts and EFB charts) have a significant impact on pilots' workload. This is an important statistical test for this study as it decides whether the use of EFB as part of automation contributes to the influence in the pilot workload. The researcher expected to find a significant main effect for display, but the main effect of the display from the results was not significant. As shown in Figure 3, even though the average workload obtained using EFB was higher than the paper, it was not significant. Thus, it suggests that neither the use of paper nor EFB charts have a significant impact on the pilot workload. The following will discuss the possible reasons for this.

Sample size. The reduced level of power in the study is one of the primary factors for the result. The observed power for this test was .059. This means that based on the sample size of 16 participants, there was only a $0.59 \%$ chance of 
deducting a difference in pilot workload scores for display. This means that recruiting a larger sample size could have increased the power, and hence it would have made the test significant.

Regression towards the mean. The secondary factor that could have been a threat to internal validity is the regression towards the mean effect (Privitera, 2017). This could have occurred from the participants' improvement by flying the second to a level closer to the mean of the participants' actual ability. For example, if the participant flew a conventional approach using the EFB chart earlier, and in the next scenario he or she flew the same unexpected scenario with an EFB, the participant would have flown the second scenario using the EFB to his or her true potential level than before. This, in return, would have caused the participant's ability to manage the demanding tasks at a level closer to their actual ability.

Testing effect. The third factor is the testing effect (Privitera, 2017). During the study, the participants progressed from one scenario to another immediately after completing the NASA-TLX assessment. Therefore, the retainment of knowledge from the previous scenario might have improved their ability to manage the tasks better in the next scenario. For example, if the participant's first attempt was to fly an unexpected scenario with paper charts and in the next scenario, he or she flies an unexpected scenario using EFB, the practice learned from the earlier scenario would have helped the participant to fly the second scenario better.

Demanding tasks. The tasks tied to the use of EFB might not be demanding enough to expose the participants to a higher level of workload. For example, in this study, both the expected and unexpected scenarios were based on VFR approaches on a clear day. Thus, it only requires the participants to use the FAA sectional charts and the airport map to locate the runway. This would have led the participants only to utilize EFB functions necessary for a VFR approach. Thus, the extensive use of other aeronautical functions in the EFB was not utilized.

\section{Interaction Between Approach and Display}

This is to test whether the levels of display alter the levels of approach. The observed power for this test was .096. This means that based on the sample size of 16 participants, there was only a $0.96 \%$ chance of deducting an interaction between approach and display. The result shows that the interaction between approach and display was not significant. This suggests that the use of EFB or paper display did not significantly influence the workload experienced by the pilot when flying either an expected or unexpected approach. The primary factor for this result continues to be the lack of statistical power in the study and the limited operational use of the EFB during the scenarios.

Familiarity with EFB software and iPad. The student pilots from ERAU are familiar with the Foreflight software and iPad used as an EFB. As such, they would have found operating the EFB manageable even in highly demanding task situations. For example, during the flight scenarios, the participants using the EFB 
were seen zooming at the sectional charts and maps with their fingers in one hand while moving the aircraft yoke with the other hand. Interestingly, this behavior was reported as a safety risk by the pilots in the ASRS reports. It could be possible that the participant's familiarity with the EFB Foreflight software and their ability to operate an iPad would have led them to a similar behavior when flying the scenarios at the laboratory.

Experimental realism. Experimental realism refers to whether the simulated environment used in the study was realistic enough to trigger the mental states of the participant, like when in the actual flight environment (Privitera, 2017). For example, the Elite-P1 135 BATD simulator is a low fidelity simulator. Unlike high fidelity simulators, it does not provide an enclosed environment of an aircraft cockpit with realistic flight instruments and communication devices. Furthermore, the absence of communication devices such as pilot headsets could have minimized the level of realism in communicating with the ATC. Overall, the reduced realism in the simulator could have influenced the measured workload in the study.

Project timeline. The first factor to consider is the limited timeline (16 weeks) that was available to complete the project. This includes the time taken to write the proposal to conduct the study and send it to the IRB, making amendments to the proposal for final approval, recruitment of participants, and scheduling them based on the availability of the simulator at the Cognitive Engineering Research in Transportation Systems laboratory. These tasks eat into the existing timeline by four to six weeks. On the other hand, a larger time frame could provide the researcher more time to recruit a larger sample size to do the study or to do a pilot study first to detect any gaps in the experiment.

\section{Conclusion}

This study indicates that the increase in task demand during an unexpected approach has a direct influence on the pilot workload. It also points out that the use of EFB does not significantly influence the pilot workload. However, the study also shows that given any increase in the task demands during unexpected situations, it may lead to a significant rise in pilot workload. The study has also shown that the influence on the pilot workload due to the difference in the visual layout of paper and EFB charts do not significantly influence the pilot workload. From the study, it can also be seen that the utility of NASA-TLX to measure pilot workload was successfully administered in a simulated environment. Its ability to address workload experienced during expected and unexpected approach provides deeper insight into how demanding tasks can influence pilot workload. The study suggests that even though the general literature about the influence of automation on workload exists, this study demonstrates that its influence is still debatable. 


\section{References}

Archer, J., Keno, H., \& Kwon, Y. (2012). Effects of automation in the aircraft cockpit environment: Skill degradation, situation awareness, workload. Purdue University, West Lafayette, ID. Retrieved from https://pdfs.semanticscholar.org/a90a/7a1dc3828eb34e79a40accf771e7c54 5445f.pdf

Ates, A. S. (2017). Electronic flight bag in the operation of airline companies: Application in Turkey. Computer Science and Information Technology, 5(4), 128-134. doi:10.13189/Csit.2017.050402.

Aviation Safety Reporting System. (2018). ASRS database online. Retrieved from https://asrs.arc.nasa.gov/search/database.html

Babb, T. A. (2017a). Electronic flight bag policies at collegiate aviation programs. International Journal of Aviation, Aeronautics, and Aerospace, 4(4). Retrieved from https://commons.erau.edu/ijaaa/vol4/iss $4 / 8$

Babb, T. A. (2017b). Professional pilot commercial off-the-shelf (COTS) EFB usage, policies and reliability. International Journal of Aviation, Aeronautics, and Aerospace, 4(1). doi:/10.15394/ijaaa.2017.1159

Cahill, J., \& Donald N. Mc. (2006). Human computer interaction method for electronic flight bag envisionment and design. Cognition, Technology \& Work, 8(2). Retrieved from https://link.springer.com/article/ 10.1007/s10111-006-0026-z

Chandra, D. C. (2003, October). A tool for structured evaluation on electronic flight bag usability. Paper presented at 22nd Digital Avionics System Conference, Indianapolis, IN. doi:10.1109/DASC.2003.1245958

Chandra, D. C., \& Kendra, A. (2009). Review of safety reports involving electronic flight bags. In 2009 International Symposium on Aviation Psychology (p. 407).

Endsley, M. R. (1999). Situation awareness in aviation systems. Handbook of aviation human factors, 257-276. Mahwah, NJ: Lawrence Erlbaum.

German, E. S., \& Rhodes, D. H. (2016). Human-model interactivity: What can be learned from the experience of pilots with the glass cockpit? Paper presented at the 2016 Conference on System Engineering Research, Massachusetts Institute of Technology, Cambridge, U.S.A. Retrieved from http://seari.mit.edu/documents/preprints/GERMAN_CSER16.pdf

Haddock, K. N., \& Beckman, W. S. (2015). The effect of electronic flight bag use on pilot performance during an instrument approach. Collegiate Aviation Review, 33(1), 1-13.

Joslin, R. (2013). Human factors hazard of iPads in general aviation cockpits. Paper presented at Human Factors and Ergonomic Society 57th annual meeting, Boston, MA. doi: 10.1177/1541931213571015 
Ohme, M. (2014). Use of tablet computers as electronic flight bags in general aviation. Retrieved from https://commons.erau.edu/aircon/

Parasuraman, R., Sheridan, T. B., \& Wickens, C. D. (2008). Situation awareness, mental workload, and trust in automation: Viable, empirically supported cognitive engineering constructs. Journal of Cognitive Engineering and Decision Making, 2(2), 140-160. doi:/10.1518/155534308X284417

Privitera, J. G. (2017). Research methods for the behavioral sciences (2nd ed.). Los Angeles, CA: SAGE.

Salas, E., \& Maurino, D. (Eds.). (2010). Human factors in aviation. London, UK: Elsevier Inc.

X Plane 11. (2019, March 5). Press kit. About us. Retrieved from https://www.Xplane.com/press-kit/ 